\title{
Weichteilschaden bei offenen und geschlossenen Frakturen
}

\author{
Hans-Joerg Oestern
}

\section{Zusammenfassung}

Die Einschätzung der Schwere des Weichteilschadens stellt einen wichtigen Faktor in der Beurteilung des Managements von offenen und geschlossenen Frakturen dar. Die Reaktion der Weichteile auf eine Verletzung betrifft mikrovaskuläre Veränderungen und Entzündungsprozesse, die ihrerseits lokal eine Gewebshypoxie und Azidose bewirken. Operative Zugänge, die diese pathophysiologischen Mechanismen nicht bedenken, können zu Gewebsnekrosen und sekundären Infektionen führen. Somit kommt den pathophysiologischen Mechanismen und der korrekten Einschätzung eines Weichteilschadens eine wesentliche operationstaktische Bedeutung zu.
Der Weichteilschaden bei einer offenen oder geschlossenen Fraktur führt zu entsprechenden Reaktionen, die phasenhaft ablaufen.

Im Allgemeinen werden 4 Phasen unterschieden:

1. Gerinnungsphase

2. Entzündungsphase

3. Granulationsphase

4. Narbenbildung
OP-JOURNAL 2009; 25: 154-159

(c) Georg Thieme Verlag KG Stuttgart · New York DOI http://dx.doi.org/10.1055/s-0029-1240663

\section{Soft Tissue Injuries in Open or Closed Fractures}

Determination of the severity of injury to the soft tissues is an important component of patient assessment and impacts on the management of closed fractures. The response of soft tissue to blunt injury involves microvascular and inflammatory processes that produce localised tissue hypoxia and acidosis. Incisions placed through such compromised tissue can lead to wound breakdown and deep infection. Therefore, recognising the signs of soft tissue injury is the foundation for a successful management of closed fractures. Many treatment options, including splinting, cryotherapy, compression, and delayed surgery, help limit further soft tissue injury and facilitate its rapid recovery before surgical intervention. Emerging surgical techniques based on improved management have resulted in decreased rates of soft tissue complications.

\section{Gerinnungsphase}

Das Ziel der Gerinnungsphase besteht in der Vermeidung eines weiteren Blutverlusts. Durch die Verletzung werden Gefäße zu einem Vasospasmus angeregt, der ca. $10 \mathrm{~s}$ anhält. Dieser Effekt hat nur einen geringen Einfluss auf die Blutstillung, da 90\% des Blutverlusts aus Kapillaren stammt, die über keine Gefäßmuskulatur verfügen. Maßgeblich für die Gerinnungsprozesse sind die Thrombozyten, die an der Gefäßwand haften bleiben. Dieser Haftungsprozess wird durch das an Thrombozytenmembran gebundene Glykoprotein Ib-1X-V initiert. Durch die aktiven Aggregationsrezeptoren auf der Thrombozytenoberfläche können sich die Fibrinfilamente des Blutstroms mit den Thrombozyten vernetzen und auf der Gefäßläsion eine Art Landeplatz für nachfolgende weiße Blutplättchen bilden. Der anfängliche Verschluss aus Thrombozyten wird mit einem Fibrinnetz stabilisiert. Das entscheidende $\mathrm{Ge}$ rinnungsenzym ist das Thrombin, das aus dem Gerinnungssystem Fibrin freisetzt, welches dann wiederum von anderen Enzymen miteinander vernetzt wird [8]. Durch Aktivierung des Thrombins läuft eine enzymatische Kettenreaktion $\mathrm{ab}$, wobei durch weitere Faktoren eine 100000 -fache Beschleunigung der enzymatischen Reaktionskette erreicht wird. Dabei sind einige enzymatische Reaktionen an die vor Ort befindlichen Zellmembranen gebunden, um das System lokal begrenzt zu gestalten. Die sich daran anschließende Entzündungsphase ist durch eine Infiltration der Wundränder mit Leukozyten (Granulozyten, Lymphozyten) und Monozyten charakterisiert. Die Leukozyten werden durch Chemotaxine angelockt, die von Gewebsnekrosen, Bakterien und Blutbestandteilen freigesetzt werden. Anschließend werden chemotaktisch neutrophile Granulozyten, Makrophagen und Lymphozyten in den Weichteilschaden angezogen. Die Reize für das Einwandern sind Zytokine wie Interleukin-1, Tumor-Nekrose-Faktor und TGF- $\beta$. Es sind wahrscheinlich mehrere Signale involviert, die bisher jedoch noch nicht alle identifiziert werden konnten. Nach dem Einwandern dieser Zellen kommt es zu einer lokalen Freisetzung von Zytokinen und Wachstumsfaktoren. Von Letzterem sind inzwischen über 400 identifiziert worden [8]. Anschließend erfolgt die Aktivierung migrierender Zellen, da nur aktivierte Zellen im Wundgebiet funktionell wirksam sind und zu einer Modulation der Wundheilungskaskade führen können. Die Zellen, die in das Wundgebiet migrieren, haben eine unterschiedliche Funktion. Makrophagen sind für die Phagozytose, Débridement, Matrixsynthese, Zellaktivierung und Angiogenese zuständig, Fibroblasten sind verantwortlich für die Kollagensynthese, Lymphozyten für die humerale Abwehr 
und neutrophile Granulozyten zur Abwehr bei bakteriellen Kontaminationen. Von allen beteiligten Zellen haben jedoch die Makrophagen die wichtigste Funktion.

\section{Frakturheilung}

In gleicher Weise läuft die Frakturheilung ab, bei der ebenfalls lokale und systemische Prozesse bestimmend sind. Auch hier sind lokale, molekulare und zelluläre Abläufe bestimmend. Ebenso gibt es Hinweise für eine systemische Freisetzung mesenchymaler Stammzellen im Frakturbereich. Extrinsische Faktoren wie Medikamente und Alter beeinflussen die lokalen Prozesse und verändern das Outcome der Frakturen, während die Biomechanik der Frakturstabilisierung die Biologie der Frakturheilung beeinflusst.

Die Regeneration des Knochens hängt von 3 wesentlichen Elementen ab:

1. von den Vorläuferzellen

2. den Wachstumsfaktoren (Osteoinduktion)

3. von einer adäquaten Umgebung (Osteokonduktion)

Hypoxie beeinflusst die osteogene Differenzierung von menschlichen mesenchymalen Stammzellen durch Reduzierung der Zahl lebender Zellen und durch Veränderung der molekularen Signale [6, 7].

Man glaubt heute, dass mesenchymale Stammzellen eine trophische Aktivität in den frühen Stadien der Frakturheilung ausüben. Mesenchymale Stammzellen werden lokal freigesetzt vom Knochenmark, Periost, dem kortikalen Knochen und vor allen Dingen dem umgebenden Weichteilgewebe.

Die Anwesenheit adulter Stammzellen in der Muskulatur wird durch 2 Beobachtungen unterstützt:

1. Muskulatur kann sich in Knochen umwandeln, z. B. bei heterotoper Ossifikation.

2. In der Originalbeschreibung der Osteoinduktion durch Urist wurden die Effekte von BMP auf Vorläuferzellen innerhalb des Muskelgewebes beschrieben.

Die Bedeutung der Muskulatur als Quelle von Osteoprogenitorzellen wird unterstrichen durch die schlechte knöcherne Heilung bei offenen Frakturen mit schwerem Muskeltrauma.

\section{Definition der Wunde}

Die Definition der Wunde lautet: Eine mehr oder weniger klaffende Gewebedurchtrennung der äußeren Haut, der Schleimhäute und der Oberfläche von Organen.

An der Haut unterscheiden wir eine oberflächliche Schürfwunde, die auf die Epidermis beschränkt ist und durch oberflächlich tangential wirkende Kräfte entsteht. Eine tiefere Schürfwunde bedeutet eine Beteiligung des Coriums durch eine stärker wirkende Gewalteinwirkung.

Die Kontusion entsteht durch Übertragung der kinetischen Energie beim $\mathrm{Zu}$ sammenprall und umfasst die Haut und unter Umständen auch tiefer liegende Strukturen. Es kommt zu einer Verletzung von Gefäßen und zu Einblutungen unter der Haut.

Eine Ablederungswunde (Décollement) entsteht aufgrund einer tangentialen Gewalteinwirkung, wobei Haut und Unterhaut von der resistenteren Unterlage abgestreift werden. Auf diese Weise bilden sich unter Umständen flächenhafte Hämatome.

Als Zertrümmerung bzw. Zermalmung (Conquassatio) bezeichnen wir eine hochgradige mechanische Zerstörung von Geweben, Organen oder Körperteilen. Häufig kann jedoch von der äußerlichen Inspektion keine definitive Klassifizierung eines Weichteilschadens vorgenommen werden. Eine Hilfe kann unter Umständen das Röntgenbild liefern.

\section{Weichteilschaden und Röntgenbefund}

Ausgedehnte Dislokation, Knochenzertrümmerung sowie röntgenologisch sichtbare Veränderungen der Weichteile (Fremdkörper- und Lufteinsprengungen, Geschosse, Weichteildefekte) können bereits einen Hinweis auf das Schwerebild der Weichteilschädigung geben.

Andererseits kann jedoch auch eine einfache Frakturform von einem ausgedehnten Weichteilschaden begleitet sein, da die Spontanreposition oder die Einrichtung des Bruches am Unfallort zu einer falschen Deutung des primären Röntgenbefunds führen kann. Glatte Querfrakturen oder auch 2-Etagen-Brüche am Unterschenkel sind jedoch $\mathrm{zu}^{-}$ meist Folge eines direkten Traumas und damit durch einen entsprechenden
Weichteilschaden charakterisiert. Mehretagenbrüche haben eine schlechte Prognose hinsichtlich der Frakturheilung.

\section{Weichteilschaden bei geschlossenen Frakturen}

Als besonders problematisch erweist sich der Weichteilschaden bei den geschlossenen Brüchen, dessen Erfassung vielfach schwieriger ist, sodass er unterschätzt oder sogar ignoriert wird. Schon eine einfache Hautkontusion einer geschlossenen Fraktur kann differenziertere therapeutische und prognostische Probleme aufwerfen als die Hautdurchspießung einer offenen Fraktur. Die Hauptkomplikation dieser Hautkontusionen ist die Nekrose, die damit einen Infektionsweg bahnt. In gleicher Weise kann die Kontamination einer tiefen Schürfung zu einer Infektion beitragen. In diesem Fall ist die natürliche Hautbarriere gegenüber einer Infektion erheblich geschwächt. Bei den offenen Brüchen wird das Ausmaß der Weichteilschädigung noch durch zusätzliche Faktoren bestimmt. Außer dem Schweregrad der knöchernen Verletzung, dem Verletzungsmechanismus und der Zeit, die vom Unfall bis zur Versorgung der Weichteile und der Fraktur entsteht, entscheidet der Grad der Kontamination über Verlauf und Prognose der offenen Fraktur.

\section{Klassifikation des Weichteilschadens}

Ziel einer Klassifizierung von Weichteilen muss es sein, möglichst umfassend $\mathrm{zu}$ beschreiben und $\mathrm{zu}$ graduieren, um eine Hilfestellung für die einzuschlagende operative Therapie zu leisten. Darüber hinaus dient sie auch der Vergleichbarkeit der Resultate und natürlich zur internen und externen Qualitätskontrolle. Darüber hinaus dient sie der Vergleichbarkeit bei gesundheitsökonomischen Berechnungen.

\section{Bisherige Einteilungen des Weichteilschadens}

Anerkannt ist lediglich die Fraktureinteilung geschlossener und offener Brüche. Während für die geschlossenen Frakturen nur eine Einteilung zur Verfügung steht, werden die offenen Frakturen nach unterschiedlichen Gesichtspunkten beurteilt. Allgöwer [1] teilte im Jahr 1971 die offenen Brüche in 1., 2. und 3. Grad ein. Eine erstgradig offene Fraktur umfasst eine Durchspießung von innen, die 

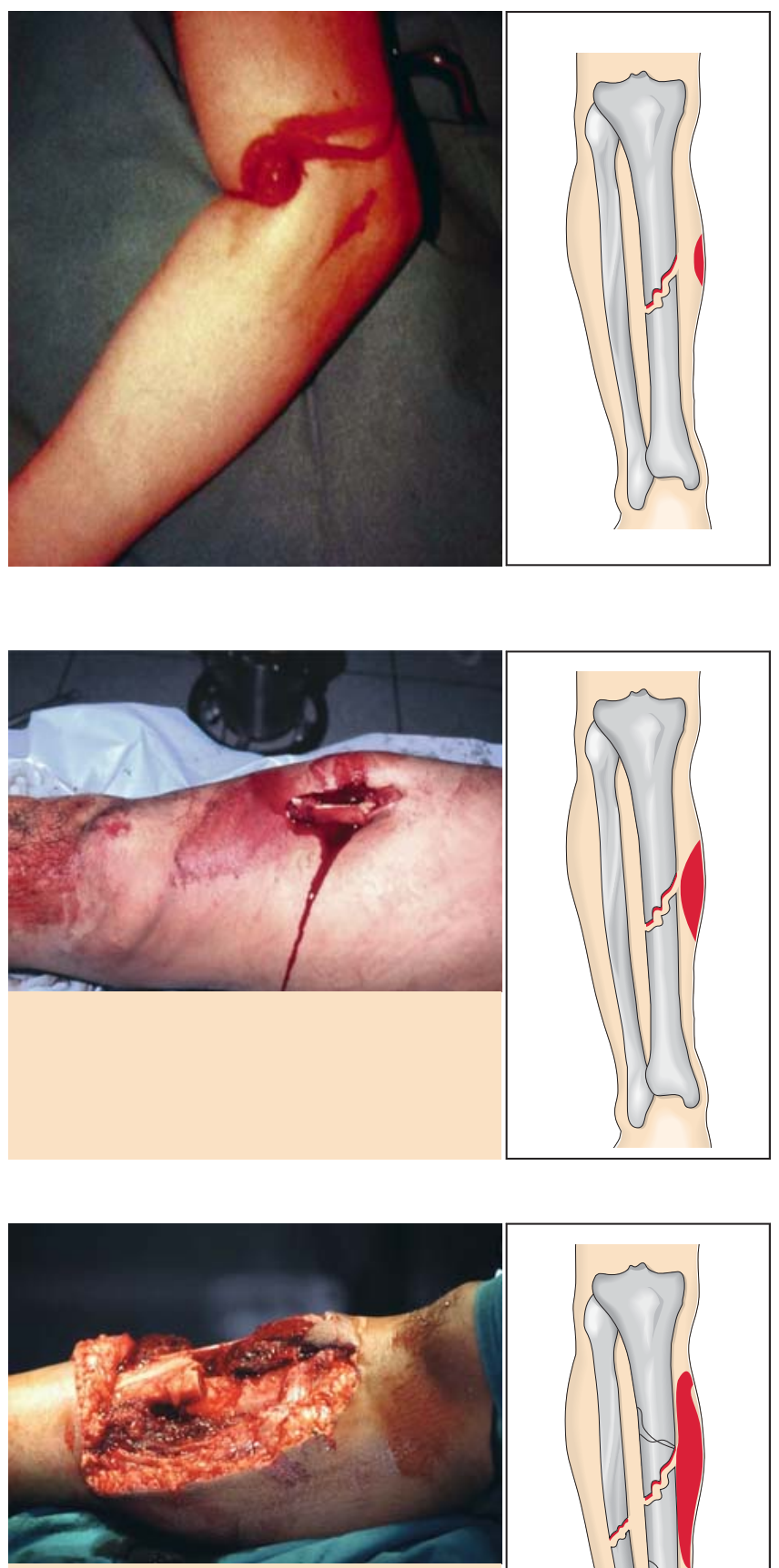

zweitgradig offene Fraktur beinhaltet die Gewebekontusion durch direkte Gewalteinwirkungen und die drittgradig offene Fraktur wird charakterisiert durch eine ausgedehnte Zerstörung von Haut, Muskulatur, Gefäßen, Nerven und Sehnen. Gustilo und Anderson [5] klassifizierten die offene Fraktur in 3 verschiedene Schweregrade, wobei das Ausmaß der Hautverletzung zugrunde gelegt wird. Grad 1 umfasst eine offene Fraktur mit
Abb. 1 Einteilung der offenen Fraktur nach Gustilo und Anderson: Die Hautwunde ist kleiner als $1 \mathrm{~cm}$.

Abb. 2 Grad 2 nach Gustilo und Anderson: Die Länge der Hautläsion ist größer als $1 \mathrm{~cm}$.

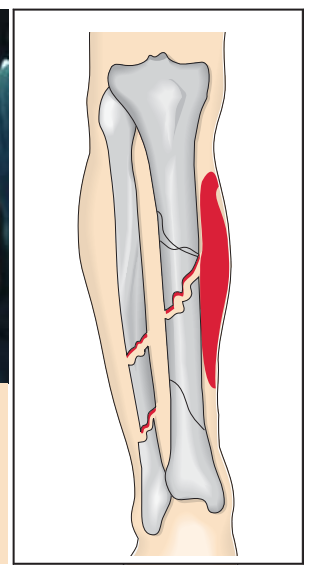

Abb. 3 Grad 3a nach Gustilo: Weichteilschaden, der noch durch lokale Maßnahmen gedeckt werden kann.

einer Wunde, die kleiner ist als $1 \mathrm{~cm}$ (Abb. 1), Grad 2 beinhaltet eine Fraktur mit einer Hautverletzung, die größer als $1 \mathrm{~cm}$ ist, aber ohne ausgedehnten Weichteilschaden (Abb. 2), die Grad-3-Fraktur umfasst eine offene Segmentfraktur sowie eine offene Fraktur mit ausgedehntem Weichteilschaden oder eine traumatische Amputation. Die Grad-3-Frakturen wurden dann später noch unterteilt in $3 \mathrm{a}$ (Abb. 3), 3 b und $3 \mathrm{c}$ (Abb. 6). Dabei beinhaltet $3 \mathrm{a}$ die Frakturen, deren Weichteilschaden noch durch lokale Maßnahmen gedeckt werden kann. Die Grad-3b-Frakturen sind Frakturen, die nur durch Lappenplastiken gedeckt werden können (Abb.4). Die Grad-3 c-Frakturen umfassen die Frakturen mit Gefäßverletzungen (Abb. 5).

Auch die Einteilung von Cauchoix und Mitarbeitern [3,4] basiert im Wesentlichen auf der Größe der Hautwunde. Differenzierter ist die Einteilung von Anderson [2], der die offenen Frakturen nach dem Ausmaß von avaskulärem und devitalisiertem Gewebe sowie dem Fremdmaterial in der Wunde differenziert. Demnach bedeutet Typ I eine punktförmige Wunde mit geringem Weichteilschaden, Typ II eine ausgedehnte Wunde mit nur wenig avaskulärem oder devitalisiertem Weichteilgewebe und Typ III umfasst ausgedehnte Wunden mit beträchtlicher Weichteilnekrose und Fremdmaterial innerhalb der Wunde. Wesentlich komplizierter ist die Einteilung der AO, hier werden zusätzlich auch noch Sehnen- und Muskelverletzungen bewertet, sodass insgesamt 225 Möglichkeiten bestehen. Da insbesondere auch die geschlossenen Frakturen nur uniform bewertet wurden, wurde eine eigene Einteilung für geschlossene und offene Frakturen entwickelt. Demnach werden die geschlossenen Frakturen in 4 Schweregrade eingeteilt (von G0 bis G3) (Abb. 6).

\section{Geschlosssene Frakturen (Abb. 7)}

\section{Geschlossene Fraktur Grad 0 (FR.G 0)}

Hierbei findet sich keine oder nur eine unbedeutende Weichteilverletzung. Die Fraktur G0 umfasst einfache Bruchformen, d.h. Frakturen, die durch indirekten Verletzungsmechanismus entstanden sind. Ein typisches Beispiel ist die Unterschenkeldrehfraktur des Skiläufers [9].

\section{Geschlossene Fraktur Grad I (FR.G I) (Abb. 8)}

Hierbei handelt es sich um eine Fraktur mit einer oberflächlichen Schürfung oder eine Kontusion durch Fragmentdruck von innen. Die Frakturformen sind einfach bis mittelschwer. Als typisches Beispiel kann die nicht reponierte Pronations-Luxationsfraktur des OSG gelten, bei der die Weichteilschädigung vom Fragmentdruck durch die Bruchkante am Innenknöchel entsteht [9]. 

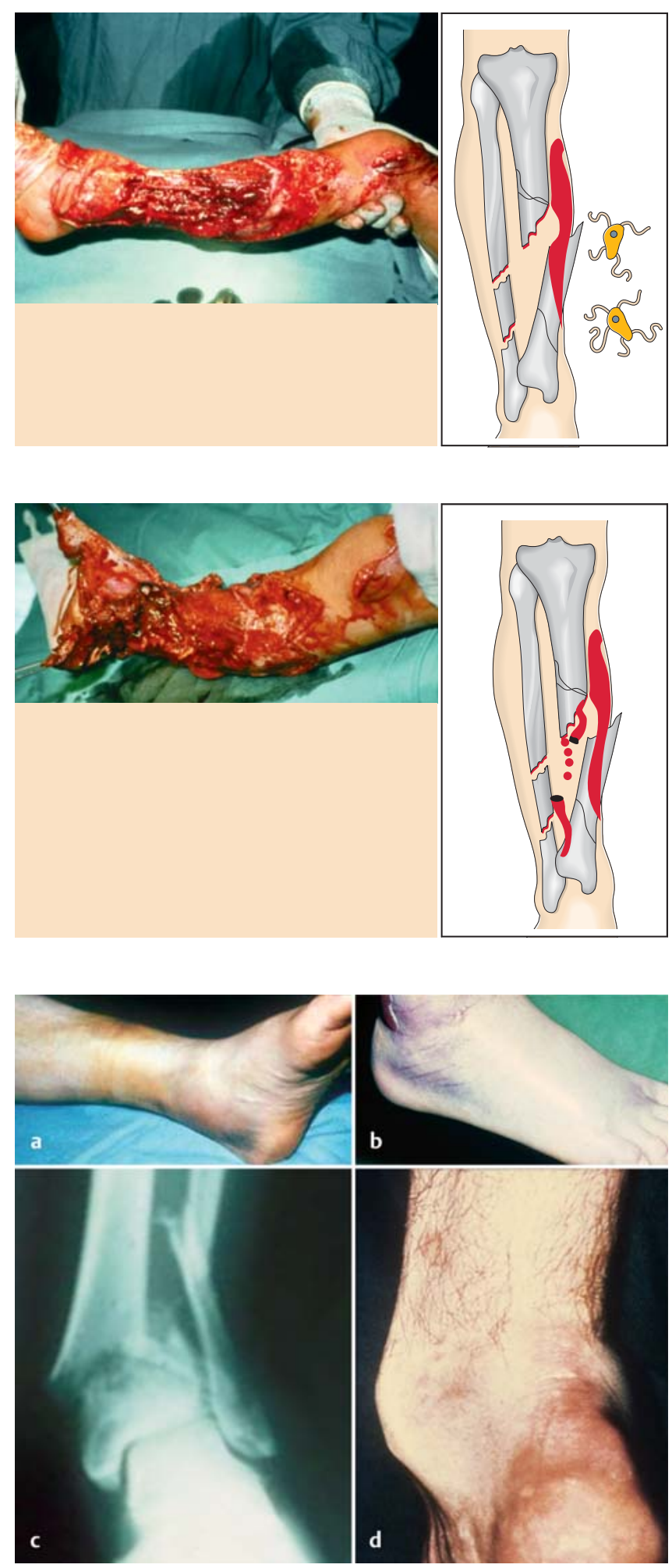

Geschlossene Fraktur Grad II (FR.G II) (Abb. 9)

Hierbei handelt es sich um eine Fraktur mit einer tief kontaminierten Schürfung sowie lokalisierter Haut- oder Muskelkontusion aufgrund eines entsprechenden direkten Traumas. Auch das drohen-
Abb. 4 Grad 3b: Offene Frakturen, die nur durch Lappenplastiken gedeckt werden können.

Abb. 5 Grad 3c: Offene Frakturen mit begleitenden Gefäßverletzungen.

Abb. 8 a bis $d$

Geschlossene Fraktur Grad I: Oberflächliche Schürfung oder Kontusion durch Fragmentdruck von innen.

Abb. 7 Geschlossene Fraktur Grad 0: Keine oder nur unbedeutende Weichteilverletzung.

destens FR.G I, meist aber FR.G II sein [9].

\section{Geschlossene Fraktur Grad III (FR.G III) (Abb. 10)}

Hierbei handelt es sich um eine Fraktur mit ausgedehnter Hautkontusion, Hautquetschung oder Zerstörung der Muskulatur, unter Umständen einem subkutanen Décollement. Ebenfalls bedeutet jedes dekompensierte Kompartmentsyndrom sowie eine Verletzung eines Hauptgefäßes bei einer geschlossenen Fraktur die Einordnung unter Grad III. Diese Gruppe umfasst schwere Bruchformen und Knochenzertrümmerungen. Durch die Quetschung der Haut und der Weichteile ist der Weichteilschaden in der Behandlung schwieriger als bei einer offenen Fraktur Grad 3 [9]. 

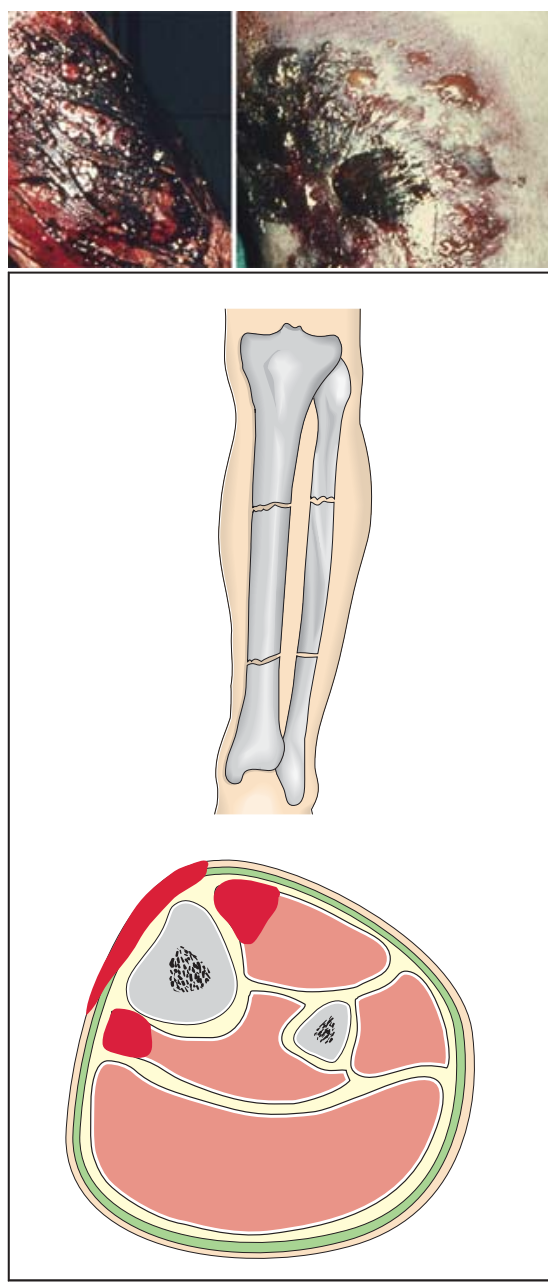

Abb. 9 Geschlossene Fraktur Grad II: Fraktur mit tiefkontaminierter Schürfung sowie lokalisierter Haut- oder Muskelkontusion.

\section{Offene Frakturen}

Die Einschätzung und Behandlung einer offenen Fraktur sollte ebenfalls an der Größe des Weichteilschadens und darüber hinaus noch an der Schwere der Kontamination ausgerichtet sein. Nicht die Größe der Hautwunde ist entscheidend, sondern der Grad der Weichteilschädigung und der Umfang der Muskelquetschung. Dies bedeutet aber auch, dass eine definitive Klassifizierung der Fraktur häufig nicht vor Behandlungsbeginn erfolgen kann. Erst durch die Freilegung der Fraktur und der Weichteile kann eine endgültige definitive Einteilung vorgenommen werden.
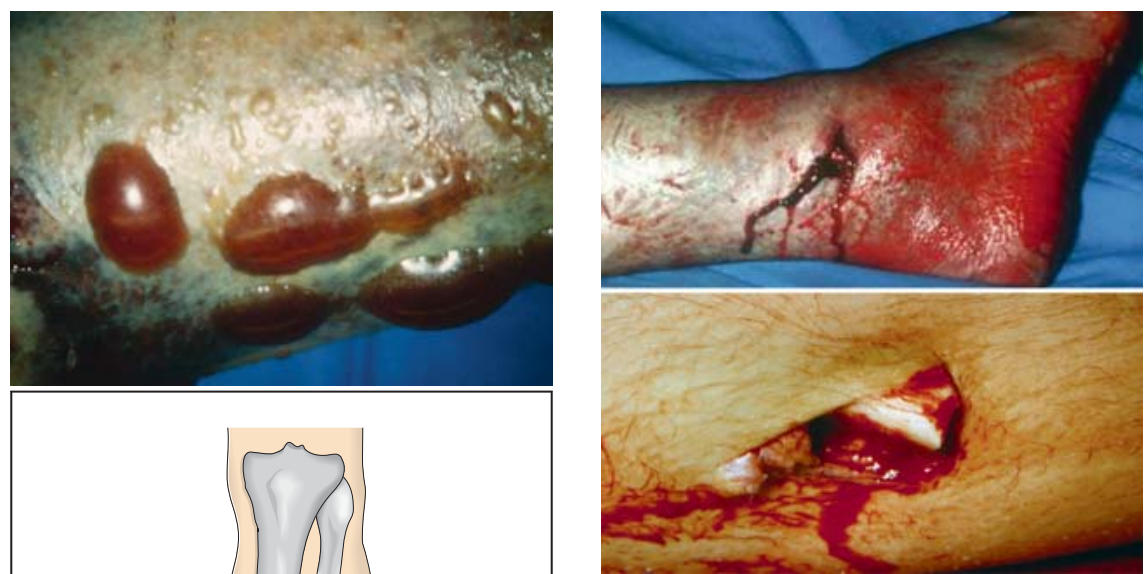

Abb.11 Offene Fraktur mit fehlender oder nur geringer Kontusion und unbedeutender bakterieller Kontamination.

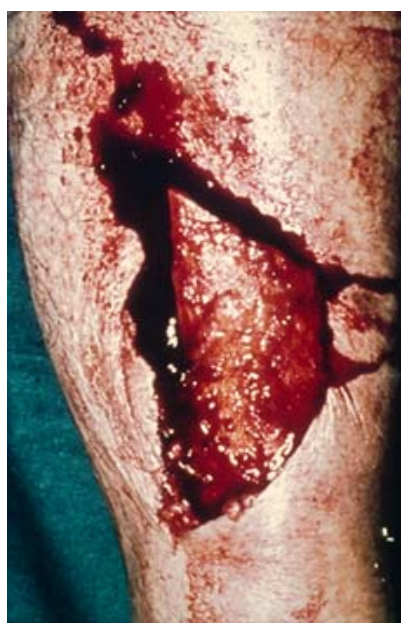

Abb. 12 Offene Fraktur Grad II: Umschriebene Haut- und Weichteilkontusion sowie mittelschwere Kontamination. Alle Frakturformen sind möglich. kontusion oder Hautquetschung (Beispiel: Kompartmentsyndrom).

\section{Offene Fraktur Grad I (FR.O I) (Abb. 11)}

Hierbei handelt es sich um eine Durchtrennung der Haut mit fehlender oder nur geringer Kontusion und einer unbedeutenden bakteriellen Kontamination. Die Haut ist gewöhnlich nur durch ein Knochenfragment unterschiedlicher Länge durchspießt. In der Regel handelt es sich um einfache Bruchformen [9].

\section{Offene Fraktur Grad II (FR.O II) (Abb. 12)}

Hierbei ist die Haut durchtrennt. Es findet sich eine umschriebene Haut- und Weichteilkontusion sowie eine mittelschwere Kontamination. Alle Frakturformen sind möglich [9]. 


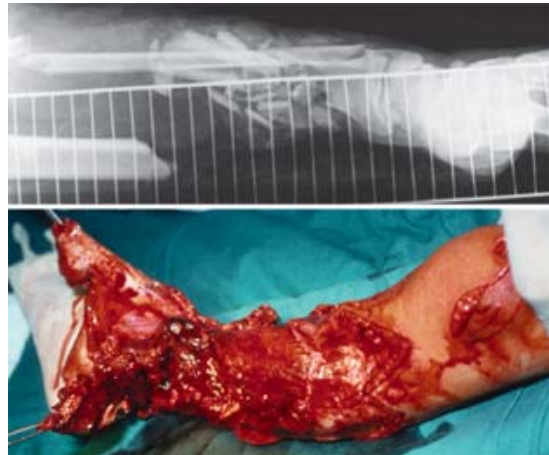

Abb. 14 Offene Fraktur Grad IV: Totale oder subtotale Amputation.

Offene Fraktur Grad III (FR.O III) (Abb. 13)

Hierbei handelt es sich um eine Hautdurchtrennung mit ausgedehnter Weichteildestruktion sowie häufig zusätzlichen Gefäß- und Nervenverletzungen. Die Wunde ist stark kontaminiert, jede offene Fraktur mit Ischämie und ausgedehnter Knochenzertrümmerung fällt unter eine Grad-III-Fraktur. Ebenso werden Schussbrüche und offene kontaminierte Frakturen bei landwirtschaftlichen Unfällen in diese Kategorie eingereiht. Aufgrund der hohen Infektgefährdung müssen alle Frakturen mit Verletzung der großen Extremitätenarterien einer offenen Fraktur Grad III zugeordnet werden [9].

\section{Offene Fraktur Grad IV (FR.O IV) (Abb. 14)}

Hierbei handelt es sich um eine totale oder subtotale Amputation. Nach der Definition ist eine subtotale Amputation durch die Durchtrennung der wichtigsten anatomischen Strukturen, besonders der Hauptgefäßverbindungen mit totaler Ischämie charakterisiert. Vom Weichteilmantel darf nicht mehr als maximal 1/4 der Zirkumferenz erhalten sein. Bestehen noch wesentliche anatomische Verbindungen und deutliche Zeichen einer Restdurchblutung - sogenannte Revaskularisation -, so kann man nur von einer offenen Fraktur Grad III sprechen. Auf eine weitere Unterteilung der offenen Fraktur Grad IV, die für die Replantationschirurgie notwendig ist (MakroMikro-Replantation, Zustand des Amputats, Ischämiezeit, Begleitverletzungen etc.) sowie die differenzierteren Unterteilungen der subtotalen und totalen Amputation wurde aus Gründen der Übersichtlichkeiten unserer Einteilung des Weichteilschadens verzichtet [9].

\section{Literatur}

${ }^{1}$ Allgöwer M. Weichteilprobleme und Infektrisiko der Osteosynthese. Langenbecks Arch Chir 1971; 329: 1127-1136

2 Anderson LD. Fractures. In: Crenshaw AHC. Campbells operative orthopaedics. St. Louis: Mosby; 1971

${ }^{3}$ Cauchoix J, Lagneau P, Boulez P. Traitement des fractures ouvertes de jambe. Resultats de 234 cas observés entre le $1^{\mathrm{er}}$ janvier 1955 et le 12 juin 1964. Ann Chir 1965; 19: 1520 1539

${ }^{4}$ Cauchoix J, Duparc J, Boulez P. Traitement des fractures ouvertes de jambe. Med Acta Chir 1975; 83: 811-822

${ }^{5}$ Gustilo B, Anderson JP. Prevention of infection in the treatment of one thousand and twenty-five open fractures of long bones. J Bone Joint Surg [Am] 1976; 58: 453-458

${ }^{6}$ Hohn DC, MacKav RD, Halliday B et al. Effect of $\mathrm{O}_{2}$ tension and microbicidal function of leukocytes in wounds and in vitro. Surg Forum 1976; 27: 18-20

${ }^{7}$ Hunt TK, Linsey M, Grislis G et al. The effect of different ambient oxygen tensions on wound infection. Ann Surg 1975; 181: 35-39

${ }^{8}$ Nerlich M, Berger A. Weichteilverletzungen und -infektionen. Heidelberg: Springer; 2002

9 Oestern H-J, Tscherne H. Pathophysiologie und Klassifikation des Weichteilschadens bei Frakturen. Orthopäde 1983; 12: 2-8

\section{Prof. Dr. med. Hans-Joerg Oestern} Chefarzt

Klinik für Unfallchirurgie, Orthopädie und Neurotraumatologie Allgemeines Krankenhaus Celle Siemensplatz 4 29223 Celle

hans-joerg.oestern@akh-celle.de 